\title{
Sexual activity as a trigger of myocardial infarction. A case-crossover analysis in the Stockholm Heart Epidemiology Programme (SHEEP)
}

\author{
J Möller, A Ahlbom, J Hulting, F Diderichsen, U de Faire, C Reuterwall, J Hallqvist
}

\begin{abstract}
Objective-To investigate sexual activity as a trigger of myocardial infarction and the potential effect modification of physical fitness.

Design-A case-crossover study nested in the Stockholm Heart Epidemiology Programme (SHEEP).

Setting-Stockholm County from April 1993 to December 1994.

Patients-All patients with a first episode of non-fatal acute myocardial infarction admitted to coronary care units were eligible, and 699 patients participated in an interview.

Main outcome measures-Relative risks with $95 \%$ confidence intervals.

Results-Only $1.3 \%$ of the patients without premonitory symptoms had sexual activity during two hours before the onset of myocardial infarction. The relative risk of myocardial infarction was 2.1 (95\% confidence interval (CI) 0.7 to 6.5 ) during one hour after sexual activity, and the risk among patients with a sedentary life was 4.4 (95\% CI 1.5 to 12.9$)$.

Conclusions-The increased risk of myocardial infarction after sexual activity and the further increase in risk among the less physically fit support the hypothesis of causal triggering by sexual activity. However, the absolute risk per hour is very low, and exposure is relatively infrequent. Thus having sex once a week only increases the annual risk of myocardial infarction slightly. Counselling should focus on encouraging patients to live a physically active life and not on abstaining from sexual activity.

(Heart 2001;86:387-390)
\end{abstract}

Keywords: myocardial infarction; sexual activity

Department of Public

Health Sciences,

Division of Social

Medicine, Karolinska

Institutet, Norrbacka,

SE-171 76 Stockholm,

Sweden

J Möller

F Diderichsen

J Hallqvist

Institute of

Environmental

Medicine, Division of

Epidemiology,

Karolinska Institutet

A Ahlbom

C Reuterwall

Department of

Cardiology, Söder

Hospital, Stockholm,

Sweden

J Hulting

Institute of

Environmental

Medicine, Division of

Cardiovascular

Epidemiology,

Karolinska Institutet

U de Faire

Correspondence to:

J Möller

jette.moller@phs.ki.se

Accepted 24 May 2001
The issue of whether sexual activity might trigger an infarct is important when counselling patients at risk of acute coronary disease. Such patients often have preconceived ideas about the risks involved in having sex. ${ }^{12}$ Until recently no epidemiological study has been able to make a valid estimate of the relative risk with the aid of control data. The advice physicians give, if they give any at all, has therefore been based largely on case reports and on indirect conclusions drawn from studies of the physiological strain on the heart during sexual activity. ${ }^{2-8}$

The new case-crossover design has proved to be an important tool in studying the triggering of disease, offering more reliable evidence about causality and a better quantitative estimation of the risk of disease. ${ }^{9}$ In the first case-crossover study of sexual activity and myocardial infarction, Muller and colleagues reported a relative risk of 2.5 (95\% confidence interval (CI) 1.7 to 3.7 ) during two hours after sexual activity, and a further increase in risk among physically inactive persons..$^{111}$ However, the increase in absolute risk was very small and this has important implications for any clinical and public health advice that might be given. This is the second case-crossover study on this topic. Its objectives were to analyse whether sexual activity might trigger the onset of myocardial infarction and whether the effect is modified by physical fitness, and also to quantify the increase in risk.

\section{Methods}

The Swedish onset study is nested in the Stockholm Heart Epidemiology Programme (SHEEP), a population based case-control study of causes of myocardial infarction. The study base includes all Swedish citizens $45-70$ years of age living in Stockholm County, with no previous myocardial infarction. ${ }^{12}{ }^{13}$

The study has a case-crossover design. The development of this new epidemiological study design was inspired by " $\mathrm{N}$ of 1 " trials, in which a patient serves as his or her own control when crossing back and forth between periods of treatment and placebo. ${ }^{14}$ The case-crossover design is also based on within individual comparisons, although mostly not randomised. The starting point is that most people in their daily life cross over between short periods of exposure to hypothetical triggers and much longer periods of unexposed time. Three types of information are needed: the time of disease onset; knowledge of whether the trigger was present during a defined period immediately before onset; and the usual frequency of trigger exposure (fig 1). The "hazard period" is the period before disease onset during which the trigger has an effect. Whether a case is exposed to the trigger or not during the hazard period determines the case information and makes it possible to calculate the observed exposure odds. The control information consists of expected exposure odds, which are based on the proportion of exposed person-time for each patient (the usual frequency) during a 


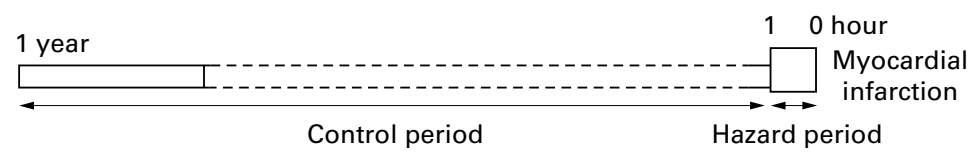

Figure 1 Illustration of the "usual frequency approach" in the case-crossover design.

defined period. By pooling individually matched comparisons a relative risk can be calculated. The relative risk measures the probability of the onset of the event during a period of exposure relative to a period of nonexposure.

The study population consisted of all patients with a first event of non-fatal acute myocardial infarction in the SHEEP study base from April 1993 to December 1994 who was admitted to one of the coronary care units in Stockholm County's hospitals. In all, 1489 patients with first event myocardial infarcts were eligible in SHEEP during this period, and of these persons, 699 were interviewed. A majority of the 790 patients who were not interviewed (495) had died or were too sick to be contacted for the Onset study. Myocardial infarction was diagnosed according to the criteria developed by the Swedish Society of Cardiology in 1991. After the exclusion of cases with missing, unreliable, or inaccurate information regarding time of disease onset or exposure, 659 cases remained. Some relevant characteristics of the Onset study population are shown in table 1. Details concerning the risk factor assessment are reported elsewhere. $^{12} 13$

Information was obtained by interviews conducted by specially trained nurses during the hospital stay or soon after discharge. Each interviewer obtained information on all episodes of pain (time, type, duration, and so on) and other symptoms and circumstances during the four days before myocardial infarction in order to determine the precise time of disease onset. The start of diagnostic chest pain was used as a marker of disease onset. Premonitory symptoms were defined as any symptoms fulfilling the criteria of a diagnostic symptom experienced over the four day period before the diagnosed onset of disease.

Table 1 Characteristics of the study population

\begin{tabular}{|c|c|c|c|c|}
\hline Characteristic & $\begin{array}{l}\text { All interviewed } \\
(n=699)\end{array}$ & $\begin{array}{l}\text { All, high } \\
\text { quality } \\
(n=659)\end{array}$ & $\begin{array}{l}\text { Without } \\
\text { premonitory } \\
\text { symptoms } \\
(n=399)\end{array}$ & $\begin{array}{l}\text { With } \\
\text { premonitory } \\
\text { symptoms } \\
(n=260)\end{array}$ \\
\hline Male & 49.9 & 49.5 & 48.9 & 50.4 \\
\hline $45-60$ years & 77.2 & 76.8 & 78.2 & 74.6 \\
\hline Married & 76.3 & 76.3 & 78.2 & 74.2 \\
\hline \multicolumn{5}{|c|}{ Socioeconomic group } \\
\hline Manual worker & 28.6 & 28.6 & 29.4 & 27.2 \\
\hline Non-manual & 60.6 & 60.3 & 59.7 & 61.2 \\
\hline Self-employed & 10.8 & 11.1 & 10.9 & 11.6 \\
\hline Hypertension ${ }^{\star}$ & 31.7 & 31.3 & 30.3 & 32.9 \\
\hline Diabetes mellitus & 14.5 & 13.7 & 12.7 & 15.4 \\
\hline \multicolumn{5}{|l|}{ Smoking† } \\
\hline Never & 24.3 & 25.0 & 24.0 & 26.4 \\
\hline Former & 28.4 & 28.5 & 28.4 & 28.6 \\
\hline Current & 47.2 & 46.6 & 47.5 & 45.0 \\
\hline Obesity & 43.3 & 43.2 & 42.8 & 43.8 \\
\hline Physically inactive & 53.2 & 52.5 & 51.8 & 53.9 \\
\hline
\end{tabular}

Values are percentages.

${ }^{\star}$ Hypertension defined as systolic pressure $\geqslant 170 \mathrm{~mm} \mathrm{Hg}$ or diastolic pressure $\geqslant 95 \mathrm{~mm} \mathrm{Hg}$. tFormer smoker defined as having stopped smoking more than two years ago.

$\ddagger$ Obesity defined as a body mass index of $\geqslant 27$.
Exposure to sexual activity was identified in the interview by the question: "When was the last time before your infarction that you engaged in sexual activity?". Patients could state their response in terms of how many minutes, hours, days, weeks, months, or years ago the activity took place. The usual annual frequency was assessed through the question "How often?", allowing the patient to answer in terms of number of times a day, week, month, or year. Patients having sex more than once a month were asked an additional question: "At what time during the day do you usually engage in sexual intercourse?". In order to obtain information on data quality, interviewers made a judgement about and noted whether or not the questions regarding sexual activity seemed to embarrass the patient.

Physical fitness was determined by questions on the frequency and intensity of the usual level of physical exercise. Such questions were asked in the SHEEP questionnaire (answered by $91 \%$ of the Onset cases). For the analysis of effect modification, patients were placed in two categories: "very little exercise" or "isolated walks", and "occasional exercise" or "regular exercise (at least once a week)". Other potential triggers were assessed through the interview as described in earlier published papers. ${ }^{15} 16$

\section{STATISTICAL ANALYSIS}

An episode of sexual activity was assumed to have a short duration and to occur infrequently enough for hazard periods not to overlap. Standard Mantel-Haenszel methods for follow up studies with sparse data in each stratum were used for the statistical analysis. ${ }^{17-19}$ The effect was measured as a relative risk, estimated by the ratio between the observed exposure odds at the time of disease onset and the expected exposure odds. Through stratification by potential effect modification of physical fitness, group specific relative risks were assessed, and differences between groups were examined by $\chi^{2}$ tests. ${ }^{20}$ Calculations were made using SAS version 6.12 and Microsoft Excel 7.0.

\section{Results}

Premonitory symptoms were experienced by $39.5 \%$ of the patients (260/659). As the premonitory symptoms might lead to a biased estimation of the relative risk, our main analyses were restricted to the group of patients who had not had any premonitory symptoms. None of the patients with experience of a premonitory symptom had been engaged in sexual activity during the four hours before their infarction.

Among patients who did not have any premonitory symptoms, $1.3 \%$ (5/399) were exposed to sexual activity during the two hours before their infarction. During the hour directly before the infarct (0-60 minutes) a raised point estimate of 2.1 ( $95 \%$ CI 0.7 to 6.5 ) was found, and during the hazard period before that hour (61-120 minutes) a risk of 1.4 (95\% CI 0.3 to 5.8 ) remained (table 2). The point estimate of the relative risk during the total two hour hazard period was 1.8 (95\% CI 0.7 to 4.3) (table 2). In the analyses of all cases, the 
Table 2 Relative risk of myocardial infarction after an episode of sexual activity

\begin{tabular}{lllll}
\hline $\begin{array}{l}\text { Hazard period } \\
\text { (min) }\end{array}$ & $\begin{array}{l}\text { Number of } \\
\text { exposed cases } \\
(n=659)\end{array}$ & $\begin{array}{l}\text { Relative risk } \\
\text { (95\% CI) }\end{array}$ & $\begin{array}{l}\text { Number of exposed cases } \\
\text { among those with no } \\
\text { premonitory symptoms } \\
(n=399)\end{array}$ & $\begin{array}{l}\text { Relative risk } \\
\text { (95\% CI) }\end{array}$ \\
\hline $0-60$ & 3 & $1.4(0.4$ to 4.2$)$ & 3 & $2.1(0.7$ to 6.5$)$ \\
$60-120$ & 2 & $0.9(0.2$ to 3.7$)$ & 2 & $1.4(0.3$ to 5.8$)$ \\
$120-180$ & 0 & $0.5(0.1$ to 3.3$)$ & 1 & $0.7(0.1$ to 5.1$)$ \\
$180-240$ & 1 & $1.1(0.5$ to 2.8$)$ & 5 & $1.8(0.7$ to 4.3$)$ \\
$0-120$ & 5 & $0.7(0.3$ to 1.8$)$ & 5 & $1.1(0.5$ to 2.8$)$ \\
$0-180$ & 5 & $0.7(0.3$ to 1.5$)$ & 6 & $1.0(0.5$ to 2.3$)$ \\
$0-240$ & 6 & & &
\end{tabular}

CI, confidence interval.

increase in relative risk was less pronounced due to the lack of exposed cases in the group with premonitory symptoms.

An analysis of potential effect modification was performed for patients without premonitory symptoms and with a hazard period of two hours. The analysis showed a substantial difference in trigger risk depending on the physical exercise pattern of the patient, although this difference was not significant $\left(\chi^{2}\right.$ test: $p=0.10)$. In the group of patients who normally get very little exercise the relative risk was 4.4 (95\% CI 1.5 to 12.9 ), compared with the relative risk of 0.7 (95\% CI 0.1 to 5.1 ) for patients with a more active exercise pattern.

\section{Discussion}

The results of our study are very similar to the findings of Muller and colleagues, and support their conclusion that sexual activity is a potential trigger of myocardial infarction. ${ }^{10}$ Our studies are almost identical in their methodology. Muller and colleagues used the casecrossover study design, had similar questionnaires, and used interviews for gathering exposure information. ${ }^{10}$ There are, however, also some differences. The Stockholm study was nested in a population based case-control study, with an age span of subjects of 25 years (45-70 years), a much shorter interval than the 20-92 years in the Boston study (mean ages 59.1 and 54.9, respectively). The present study covered only first time myocardial infarcts, whereas Muller and colleagues included patients with a history of myocardial infarction. The Boston study was larger, with 1633 patients, of whom $858(48 \%)$ were sexually active during the year before the infarction, compared with 659 patients in our study, with $560(85 \%)$ being sexually active. We found $0.9 \%(5 / 560)$ of the sexually active patients were exposed during the two hour hazard period $(1.4 \%$ (5/345) among patients without premonitory symptoms). The corresponding percentage for the Boston study was 3.0. ${ }^{10}$

Muller and colleagues used frequency of physical exertion per week as a measure of physical fitness, and found that the trigger risk of sexual activities decreases with increased physical activity. ${ }^{10}$ The result of our analysis of effect modification also supports the finding of Muller and colleagues. Employing usual frequency of physical exertion from the Onset interview instead of the SHEEP questionnaire does not alter our conclusions.

The Swedish onset study was designed to study various potential triggers, not just sexual activity. ${ }^{15} 16$ Among our 699 interviewed patients we could not expect to find more than a small number of cases exposed to sexual activity before infarction. Our results support the earlier study but should be interpreted with caution because of the weakness in precision.

To avoid bias, the final analyses were restricted to patients without premonitory symptoms. If a premonitory symptom indicates the beginning of disease onset-such as plaque rupture or early coronary thrombosis-case exposure would be misclassified. The hazard period of interest would be that preceding the premonitory symptom and not the period before diagnostic chest pain. The presence of premonitory symptoms may also cause a "reverse causation bias"-patients with premonitory symptoms might abstain from the sexual activity they normally would have undertaken at this time. Accordingly, none of the patients with premonitory symptoms were engaged in sexual activity during a period of four hours before their infarcts. In future studies the role of premonitory symptoms should be considered.

The self matching feature of the casecrossover design eliminates the problem of confounding effects of stable risk factors with long induction periods. The morning peak in the incidence of myocardial infarction does not coincide with the peak of sexual activity, as the sexual activity normally took place in the evenings or nights. Therefore potential bias by time of day would not lead to an overestimation of the true risk. None of the exposed cases had been exposed to other known triggers, such as physical exertion and anger, during the same hazard period as the exposure to sexual activity. Confounding by other known triggers is therefore also excluded.

Misclassification of the control information constitutes an important validity problem in case-crossover studies. ${ }^{17}$ The reported usual frequency of sexual activity might be influenced by several factors, such as recall, estimation problems, shyness, and a desire to report what is thought to be normal. During the interview, the interviewer evaluated, and noted in the questionnaire, whether patients seemed embarrassed at having to answer questions about their sex life. Most of the patients (78\%; 545/699) did not seem to be bothered at all, and they reported on average a higher usual frequency of sexual activity than those who seemed embarrassed or somewhat disturbed. Restricting the analyses to patients who did not seem embarrassed did not substantially change our results. Overreporting the usual frequency of sexual activity would lead to an underestimation of the true relative risk. The sexually active patients in this study reported sexual activity approximately four times a month. In a recent, large descriptive survey of the sexual behaviour of Swedes it was found that in the age group 50-56 years, men on average had sexual intercourse about four times per month, and women about three times a month. ${ }^{21}$ Owing to incomplete coding routines for the interviewers there were occasional difficulties 
in distinguishing between very low annual frequencies and missing responses. This might imply some degree of under reporting and lead to an overestimation of relative risk, but to eliminate the observed relative risk entirely as much as half of all the sexual activity must on average have been unreported. This seems unlikely. Further, when we confine the analyses to patients with confident reports of the usual frequency, the relative risk did not alter.

The design eliminates the problem of control selection, but the results may still be biased by case selection. If cases exposed to sex during or shortly before their infarction were less inclined to participate in the study, participation bias would result in underestimation. We have, however, no indication that this was the case. Also, case selection caused by survival bias can appear if exposed cases have a worse or a better prognosis simply by virtue of the fact that they were exposed.

In interpreting our results from a public health perspective, it is important to consider the absolute risk of myocardial infarction. In the Stockholm area during the SHEEP study years the average risk of a first myocardial infarct for men and women in the age interval 45-70 years was $0.300 /$ million person-hours, which corresponds to an annual risk of $0.263 \%$. A man aged $56-60$ years having sex once a week increases his annual risk of myocardial infarction from $0.464 \%$ to $0.468 \%$ (an annual risk ratio of 1.01). That the increase in annual risk is so small depends on the infrequency of sexual activity and the short effect period (approximately two hours). The results of the Boston study suggest a lower trigger risk for women than for men. An analysis stratified by sex was not possible in our study due to lack of statistical power.

Even among people with a raised baseline risk, such as those who had had an earlier myocardial infarct, the increase in risk on a yearly basis is not at all large. In SHEEP, the risk of reinfarction within one year was $10.5 \%$ among patients surviving the first 28 days after the index infarction. If these patients engaged in sexual activity once a week it would result in an annual risk difference of $0.24 \%$ (calculated on the basis of a relative risk of 2.9 reported for patients with a history of infarction ${ }^{10}$ ).

Thus we conclude- on the basis of the current results, the findings of the Boston study, and knowledge of the physiological mechanisms through which physical and mental stress act ${ }^{22} 23$ - that sexual activity may trigger a myocardial infarct. However, the increase in the absolute risk of myocardial infarction after sexual activity is very small. Physical exercise is one way of reducing even this small increase in risk.

The study was supported by grants from the Swedish Council for Social Research and the Swedish National Institute of Public Health.

1 Boykoff SL. Strategies for sexual counseling of patients following a myocardial infarction. Dimens Crit Care Nurs 1989;8:368-73.

2 Green AW. Sexual activity and the post myocardial infarction patient. Am Heart f 1975;89:246-52

3 Hellerstein HK, Friedman EH. Sexual activity and the postcoronary patient. Arch Intern Med 1970;125:987-99.

4 Tardif GS. Sexual activity after a myocardial infarction. Arch Phys Med Rehabil 1989;70:763-6.

5 Masini V, Romei G, Fiorella AT. Dynamic electrocardiogram in normal subjects during sexual activity. G Ital Cardiol 1980;10:1442-8.

6 Larson JL, McNaughton MW, Kennedy JW, et al. Heart rate and blood pressure responses to sexual activity and and blood pressure responses to sexual activit

7 Bohlen JG, Held JP, Sanderson MO, et al. Heart rate, rateBohlen JG, Held JP, Sanderson MO, et al. Heart rate, rate-
pressure product, and oxygen uptake during four sexual pressure product, and oxygen uptake durin
activities. Arch Intern Med 1984;144:1745-8.

8 Drory Y, Shapira I, Fisman EZ, et al. Myocardial ischemia during sexual activity in patients with coronary artery disease. Am f Cardiol 1995;15:835-7.

9 Maclure M. The case-crossover design: a method for studyng transient effects on the risk of acute events. Am f Epidemiol 1991;133:144-53.

10 Muller JE, Mittleman MA, Maclure M, et al. Triggering myocardial infarction by sexual activity. Low absolute risk and prevention by regular physical exertion. Determinants of Myocardial infarction Onset Study Investigators. $7 A M A$ 1996;275:1405-9.

11 Muller JE. Sexual activity as a trigger for cardiovascular events: what is the risk? Am $\mathcal{f}$ Cardiol $1999 ; 84: 2-5 \mathrm{~N}$.

12 Reuterwall C, Hallqvist J, Ahlbom A, et al. Higher relative, but lower absolute risks of myocardial infarction in women han in men: analysis of some major risk factors in the SHEEP study. The SHEEP Study Group. F Intern Med 1999;246:161-74.

13 Hallqvist J. Socioeconomic differences in myocardial infarction. Epidemiological analyses of causes and mechanisms Stockholm: Karolinska Institutet, 1998. [Thesis.]

14 Lois TA, Lavori PW, Bailar JC, et al. Crossover and self-controlled designs in clinical research. $N$ Engl f Med 1984;310:24-31

15 Möller J, Hallqvist J, Diderichsen F, et al. Do episodes of anger trigger myocardial infarction? A case-crossover analysis in the Stockholm Heart Epidemiology Program (SHEEP). Psychosom Med 1999;61:842-9.

16 Hallqvist J, Möller J, Ahlbom A, et al . Does heavy physical exertion trigger myocardial infarction? A case-crossover analysis nested in a population-based case-referent study. Am 7 Epidemiol 2000;151:459-67.

17 Marshall RJ, Jackson RT. Analysis of case-crossover designs. Stat Med 1993;12:2333-41.

18 Mittleman MA, Maclure M, Robins JM. Control sampling strategies for case-crossover studies. An assessment of relative efficiency. Am $\mathcal{F}$ Epidemiol 1995;142:91-8.

19 Greenland S, Robins JM. Estimation of a common effect parameter from sparse follow-up data. Biometrics 1985;41: $55-68$.

20 Rothman KJ. Modern epidemiology. Boston: Little, Brown Co, 1986 .

21 Folkhälsoinstitutet. Sex in Sweden, on sexuality habits in Sweden 1996. Stockholm: Swedish Public Health Institute, 1998 [in Swedish]

22 Muller JE, Tofler GH, Stone PH. Circadian variation and triggers of cardiovascular disease. Circulation 1989;4:73343.

23 Muller JE, Abdela GS, Nesto RW, et al. Triggers, acute risk factors and vulnerable plaques: the lexicon of a new factors and vulnerable plaques: the lexicon
frontier. 7 Am Coll Cardiol 1994;23:809-13. 